\title{
DSpace@MIT
}

\author{
MIT Open Access Articles
}

\section{On synchronization of coupled Hopf- Kuramoto oscillators with phase delays}

The MIT Faculty has made this article openly available. Please share how this access benefits you. Your story matters.

Citation: Chung, Soon-Jo, and Jean-Jacques Slotine. On Synchronization of Coupled HopfKuramoto Oscillators with Phase Delays. In 49th IEEE Conference on Decision and Control (CDC), December 15-17, 2010, Hilton Atlanta Hotel, Atlanta, GA, USA, 3181-3187. Institute of Electrical and Electronics Engineers, 2010. (C) Copyright 2010 IEEE.

As Published: http://dx.doi.org/10.1109/CDC.2010.5717962

Publisher: Institute of Electrical and Electronics Engineers

Persistent URL: http://hdl.handle.net/1721.1/81359

Version: Final published version: final published article, as it appeared in a journal, conference proceedings, or other formally published context

Terms of Use: Article is made available in accordance with the publisher's policy and may be subject to US copyright law. Please refer to the publisher's site for terms of use. 


\title{
On Synchronization of Coupled Hopf-Kuramoto Oscillators with Phase Delays
}

\author{
Soon-Jo Chung and Jean-Jacques Slotine
}

\begin{abstract}
This paper presents new methods and results on synchronization of coupled Hopf nonlinear oscillators, which are commonly used as the dynamic model of engineered central pattern generators (CPGs). On balanced graphs, any positive coupling gain is proven to induce almost global asymptotic synchronization, and a threshold value for truly global exponential synchronization is also computed. Furthermore, a hierarchical connection between coupled Hopf oscillators and Kuramoto oscillators is identified. Finally, a new result on the synchronization of Kuramoto oscillators with arbitrary timevarying heterogeneous frequencies and delays is derived.
\end{abstract}

\section{INTRODUCTION}

The central pattern generators (CPGs) of animals are neural networks that can produce coordinated patterns of rhythmic outputs without brain or local sensory inputs. Hence, CPGs are believed to reduce the computation burden of the brain, and to avoid issues of time-delay in feedback by using local computations in the spinal cord. The central controller, similar to the brain of an animal, can stabilize the vehicle or robot dynamics by commanding a reduced number of variables such as the frequency and phase difference of the oscillators instead of directly controlling multiple joints [1], [2]. Artificial CPG neural networks by using coupled Hopf oscillators have been demonstrated to effectively control robot locomotion [2], swimming [3], and flapping flight [4]. In addition, phase-synchronized Hopf oscillators can be applied to a synchronization model of multi-agent coordination that is governed by both the position and phase dynamics (e.g., cyclic formation of spacecraft). We consider a supercritical Andronov-Hopf bifurcation model of $\mathbf{x}=$ $(u ; v)$ :

$$
\dot{\mathbf{x}}=\mathbf{f}(\mathbf{x}, t ; \rho)=\left(\begin{array}{c}
-\lambda / \rho^{2}\left(u^{2}+v^{2}-\rho^{2} \sigma\right) u-\omega(t) v \\
\omega(t) u-\lambda / \rho^{2}\left(u^{2}+v^{2}-\rho^{2} \sigma\right) v
\end{array}\right)
$$

with $\sigma=1$. For a positive rate of convergence $\lambda>0$, it can be easily shown that any initial trajectory $(u ; v) \neq \mathbf{0}$ exponentially converges to a circle of the radius $\rho$ rotating at the time-varying frequency $\omega(t)$ with bounded $\dot{\omega}(t)$.

The Hopf oscillator has been a popular model for engineered CPGs for robot locomotion. If $\sigma \leq 0$, bifurcation occurs and the system globally converges to the origin, which is useful for fast inhibition of oscillation. For example, [4] shows that this Hopf bifurcation can be exploited to induce

This work was supported in part by the Air Force Office of Scientific Research (AFOSR).

S.-J. Chung is with the University of Illinois at Urbana-Champaign, Urbana, IL 61801 USA s jchungeillinois.edu

J.-J. Slotine is with the Massachusetts Institute of Technology, Cambridge, MA 02139 USA jjs@mit.edu a stable transition between flapping and gliding or between walking and jumping. Also, we can simplify stability proofs by exploiting the circular symmetry of the limit cycle that permits $\mathbf{f}(\mathbf{R}(\phi) \mathbf{x}, t ; \rho)=\mathbf{R}(\phi) \mathbf{f}(\mathbf{x}, t ; \rho)$ with a rotational transformation $\mathbf{R}(\phi)$ and $\mathbf{f}(k \mathbf{x}, t ; \rho)=k \mathbf{f}(\mathbf{x}, t ; \rho / k)$. If we need a non-sinusoidal waveform generated by a non-circular limit cycle, we can simply utilize conformal mapping with an analytic function $h(z)$ without affecting the stability proofs such that $u^{\prime}+v^{\prime} j=h(u+v j), j=\sqrt{-1}$.

We present new results on the synchronization stability of coupled Andronov-Hopf oscillators by using partial contraction analysis and cyclic decomposition. Previously, [4], [5] proved that coupled Hopf oscillators globally exponentially synchronize if the coupling strength is sufficiently larger than the convergence gain of the individual Hopf oscillator. In contrast, this paper shows that coupled Hopf oscillators on a balanced graph asymptotically synchronize with any positive coupling strength from almost any initial condition. This new result can be useful if a large coupling gain, which may lead to actuator saturation in transients and increase noise in the system, should be avoided especially when the desired convergence rate is large. We also show an interesting hierarchical connection between the radius and phase dynamics, whose phase model resembles Kuramoto oscillators [6], [7], [8], [9], [10]. By utilizing the same incremental stability analysis as in the Hopf model, we study the heterogeneous Kuramoto model with time-varying or random vectors of natural frequency and phase delays. In this paper, each angle $\theta_{k} \in[-\pi, \pi]$ belongs to a circle group so that $\theta_{k}=\left[\left(\theta_{k}+\pi\right) \bmod 2 \pi\right]-\pi$, and its $\mathrm{n}$-vector is on an n-torus, i.e., $\boldsymbol{\theta}=\left(\theta_{1} ; \theta_{2} ; \cdots ; \theta_{n}\right) \in \mathbb{T}^{n}$.

We use contraction analysis [11], [12] whose implication for incremental stability [13] is essential for an observer-like stability analysis in this paper. A differential stability analysis can be made exact, thereby yielding global convergence results on time-varying nonlinear systems.

Lemma 1: [11] Consider $\dot{\mathbf{x}}(t)=\mathbf{f}(\mathbf{x}(t), t)$ and some smooth coordinate transformation of the virtual displacement, $\delta \mathbf{z}=\boldsymbol{\Theta}(\mathbf{x}, t) \delta \mathbf{x}$. A region (open connected set) of the state space is called a contraction region with respect to (w.r.t.) a uniformly positive definite metric $\mathbf{M}(\mathbf{x}, t)=$ $\boldsymbol{\Theta}^{T} \boldsymbol{\Theta}$, if the generalized Jacobian in $\delta \mathbf{z}, \mathbf{F}$, is uniformly negative definite in that region, such that, $\exists \beta>0, \mathbf{F}=$ $\left(\dot{\boldsymbol{\Theta}}(\mathbf{x}, t)+\mathbf{\Theta}(\mathbf{x}, t) \frac{\partial \mathbf{f}}{\partial \mathbf{x}}\right) \mathbf{\Theta}(\mathbf{x}, t)^{-1} \leq-\beta \mathbf{I}<0$. Then, any trajectory, which starts in a ball of constant radius w.r.t. the metric $\mathbf{M}(\mathbf{x}, t)$, centered at a given trajectory and contained at all times in a contraction region w.r.t. $\mathbf{M}(\mathbf{x}, t)$, remains 
in that ball and converges exponentially to this trajectory (i.e., $\delta \mathbf{x} \rightarrow 0$ ). If the whole state space is a contraction region w.r.t. $\mathbf{M}(\mathbf{x}, t)$, the exponential convergence result is global. The convergence rate $\beta$ is the largest eigenvalue of the symmetric part of $\mathbf{F}$.

The use of a differential coordinate change $\delta \mathbf{z}=\boldsymbol{\Theta} \delta \mathbf{x}$ generalizes Krasovskii's theorem [14]. If $\mathbf{F}$ is negative semidefinite, the convergence is asymptotic (semi-contracting).

Example 1: Consider $\dot{\boldsymbol{\theta}}=-\mathbf{K}(t) \sin (\boldsymbol{\theta})$ on $\boldsymbol{\theta} \in \mathbb{T}^{n}$, where $\mathbf{K}(t)$ is a diagonal matrix. Following [11], consider $\boldsymbol{\Theta}=\operatorname{diag}\left(\frac{1}{2} \sec ^{2}(\boldsymbol{\theta} / 2)\right)$ on $\boldsymbol{\theta} \in(-\pi, \pi)^{n}$. Then, we can compute the generalized Jacobian $\mathbf{F}=\dot{\Theta} \Theta^{-1}-$ $\boldsymbol{\Theta} \mathbf{K d i a g}(\cos \boldsymbol{\theta}) \boldsymbol{\Theta}^{-1}=-\mathbf{K}$. Hence, for $\mathbf{K}(t)>0, \boldsymbol{\theta}$ tends exponentially to zero on $\boldsymbol{\theta} \in(-\pi, \pi)^{n}$ by Lemma 1 .

Often times, finding a suitable nonlinear function $\boldsymbol{\Theta}(\mathbf{x}, t)$ can be as difficult as finding a Lyapunov function candidate. We use the partial contraction lemma [12] to prove the synchronization of coupled Hopf oscillators.

Lemma 2: [12] Consider a nonlinear system of the form $\dot{\mathbf{x}}=\mathbf{f}(\mathbf{x}, \mathbf{x}, t)$. Assume that so-called the virtual system, $\dot{\mathbf{y}}=$ $\mathbf{f}(\mathbf{y}, \mathbf{x}, t)$, is contracting with respect to $\mathbf{y}$. If a particular solution of the virtual $\mathbf{y}$-system verifies a smooth specific property (e.g., a particular trajectory), then all trajectories of the original $\mathrm{x}$-system verify this property exponentially. The original system is said to be partially contracting.

Example 2: Example 1 can be proven by Lemma 2. We rewrite the system using a sinc function [7] as $\dot{\boldsymbol{\theta}}=-\mathbf{K}(t) \operatorname{diag}(\operatorname{sinc}(\boldsymbol{\theta})) \boldsymbol{\theta}$ where $\operatorname{diag}(\operatorname{sinc}(\boldsymbol{\theta}))=$ $\operatorname{diag}\left(\sin \left(\theta_{1}\right) / \theta_{1}, \cdots, \sin \left(\theta_{n}\right) / \theta_{n}\right)$. We construct an observer-like system such that $\dot{\mathbf{y}}=-\mathbf{K}(t) \operatorname{diag}(\operatorname{sinc}(\boldsymbol{\theta})) \mathbf{y}$, which has two particular solutions $\mathbf{y}=\boldsymbol{\theta}$ and $\mathbf{y}=\mathbf{0}$. The virtual displacement $\delta \mathbf{y}$ has a time-varying contraction rate $\mathbf{F}=-\mathbf{K}(t) \operatorname{diag}(\operatorname{sinc}(\boldsymbol{\theta}(t)))$, which is now independent of y. Notice $\operatorname{diag}(\operatorname{sinc}(\boldsymbol{\theta}))>0$ on $\boldsymbol{\theta} \in(-\pi, \pi)^{n}$, Hence, if $\mathbf{K}(t)>0$, we can conclude that two solutions of $\mathbf{y}$ tend exponentially fast to each other, resulting in $\boldsymbol{\theta} \rightarrow \mathbf{0}$. The present example shows that a stability analysis via partial contraction can be made much simpler than the original contraction analysis.

\section{Problem Statement}

We consider the problem of proving global phase synchronization of coupled Hopf oscillators with arbitrary phase delays and possibly different amplitudes of oscillation. The $i$-th dynamics of Hopf oscillators with diffusive couplings and phase delays on a digraph with various radii $\rho_{i} \neq \rho_{j}$ $(i=1, \cdots, n)$ can be written as

$$
\dot{\mathbf{x}}_{i}=\mathbf{f}\left(\mathbf{x}_{i}, t ; \rho_{i}\right)-k \sum_{j \in \mathcal{N}_{i}}\left(\mathbf{x}_{i}-\frac{\rho_{i}}{\rho_{j}} \mathbf{R}\left(\phi_{i j}\right) \mathbf{x}_{j}\right)
$$

where the Hopf oscillator function $f\left(\mathbf{x}_{i}, \rho_{i}\right)$ is defined in (1) and $\mathcal{N}_{i}$ denotes the set of adjacent neighbors for the $i$-th member. Also, $k$ denotes a scalar coupling strength. The matrix $\mathbf{R}$ denotes a $\mathcal{S O}(2)$ rotational matrix $\mathbf{R}\left(\phi_{i j}\right)=$ $\left[\cos \phi_{i j},-\sin \phi_{i j} ; \sin \phi_{i j}, \cos \phi_{i j}\right]$ with a constant phase difference $\phi_{i j}$ such that one cycle has $\sum \phi_{i j}=2 \pi$, and $\phi_{i k}=\phi_{i j}+\phi_{j k}$. Note that $\mathbf{x}_{i}$ can be interpreted a biased vector such that $\mathbf{x}_{i}-\mathbf{a}_{i}$ to shift the center of the limit cycle to $\mathbf{a}_{i}$.

By rewriting (2) in polar coordinates with $u_{i}=r_{i} \cos \theta_{i}$ and $v_{i}=r_{i} \sin \theta_{i}$ with a nonzero initial condition $r_{i}>0$ and $\theta_{i} \in \mathbb{T}$ such that $\theta_{i}$ is defined only on $[-\pi, \pi]$, modulo $2 \pi$, we can obtain

$$
\begin{aligned}
& \frac{d}{d t}\left(\begin{array}{c}
r_{i} \\
\theta_{i}
\end{array}\right)=\left(\begin{array}{c}
-\lambda r_{i}\left(r_{i}^{2} / \rho_{i}^{2}-1\right) \\
\omega(t)
\end{array}\right) \\
& \quad-k \sum_{j \in \mathcal{N}_{i}}\left[\left(\begin{array}{c}
r_{i} \\
0
\end{array}\right)+\frac{\rho_{i} r_{j}}{\rho_{j} r_{i}}\left(\begin{array}{c}
-r_{i} \cos \left(\theta_{i}-\theta_{j}-\phi_{i j}\right) \\
\sin \left(\theta_{i}-\theta_{j}-\phi_{i j}\right)
\end{array}\right)\right]
\end{aligned}
$$

Definition 1: Phase synchronization. The synchronization of (2) is defined as $\frac{\mathbf{x}_{1}}{\rho_{1}}=\frac{\mathbf{R}\left(\phi_{12}\right) \mathbf{x}_{2}}{\rho_{2}}=\cdots=\frac{\mathbf{R}\left(\phi_{1 n}\right) \mathbf{x}_{n}}{\rho_{n}}$ where $\phi_{1 j}$ is the phase difference between the 1 st and the $j$-th oscillator. In other words, each oscillator is attracted to a circular limit cycle with a radius $\rho_{j}$ while maintaining a prescribed phase difference with neighboring oscillators.

We first present results of the following simpler system without radii differences and phase delays, which are then generalized to (2) in Sect. III. By using $\{\mathbf{x}\}=\left(\mathbf{x}_{1} ; \cdots ; \mathbf{x}_{n}\right)$,

$$
\{\dot{\mathbf{x}}\}=\{\mathbf{f}(\mathbf{x}, t ; \rho)\}-k \mathbf{L}_{G} \otimes \mathbf{I}_{2}\{\mathbf{x}\}
$$

where $\{\mathbf{f}(\mathbf{x}, t ; \rho)\}=\left(\mathbf{f}\left(\mathbf{x}_{1}, t ; \rho\right) ; \cdots ; \mathbf{f}\left(\mathbf{x}_{n}, t ; \rho\right)\right), \otimes$ denotes the Kronecker product operation, and $\mathbf{L}_{G} \in \mathbb{R}^{n \times n}$ is the graph Laplacian defined from $\mathcal{N}_{i}$ in (2).

By setting $\rho_{i}=\rho_{j}=\rho$ and $\phi_{i j}=0, \forall i, j$, (3) reduces to $\left(\begin{array}{c}\dot{r}_{i} \\ \dot{\theta}_{i}\end{array}\right)=\left(\begin{array}{c}-\lambda r_{i}\left(r_{i}^{2} / \rho^{2}-1\right) \\ \omega(t)\end{array}\right)-k \sum_{j \in \mathcal{N}_{i}}\left(\begin{array}{c}r_{i}-r_{j} \cos \left(\theta_{i}-\theta_{j}\right) \\ r_{j} / r_{i} \sin \left(\theta_{i}-\theta_{j}\right)\end{array}\right)$

Let us rewrite (5) by using $\mathbf{r}=\left(r_{1} ; r_{2} ; \cdots ; r_{n}\right), r_{j}>0$, $j=1, \cdots, n$, and $\boldsymbol{\theta}=\left(\theta_{1} ; \theta_{2} ; \cdots ; \theta_{n}\right)$ :

$$
\begin{aligned}
& \dot{\mathbf{r}}=-\frac{\lambda}{\rho^{2}} \mathbf{D}(\mathbf{r}, \rho)(\mathbf{r}-\rho \mathbf{1})-k \mathbf{L}_{r}(\boldsymbol{\theta}) \mathbf{r} \\
& \dot{\boldsymbol{\theta}}=\omega(t) \mathbf{1}-k \mathbf{L}_{\theta}(\mathbf{r}) \sin \left(\mathbf{B}^{T} \boldsymbol{\theta}\right)
\end{aligned}
$$

where $\mathbf{D}(\mathbf{r}, \rho)=\operatorname{diag}\left(r_{1}\left(r_{1}+\rho\right), \cdots, r_{n}\left(r_{n}+\rho\right)\right),(\mathbf{r}-$ $\rho \mathbf{1})=\left(r_{1}-\rho ; \cdots ; r_{n}-\rho\right)$, and $\mathbf{1}=(1 ; 1 ; \cdots ; 1)$. Note that $\mathbf{D}(\mathbf{r}, \rho)>0$ for $\forall \mathbf{r}>0$. For $n$ oscillators with $m$ directed edges, the matrix $\mathbf{B} \in \mathbb{R}^{n \times m}$ is the incidence matrix with $\mathbf{B}_{i j}=1$ for the $j$-th edge entering the $i$-th vertex and $\mathbf{B}_{i j}=-1$ for the outgoing edge.

The nonlinear time-varying matrices $\mathbf{L}_{r}(\boldsymbol{\theta}) \in \mathbb{R}^{n \times n}$ and $\mathbf{L}_{\theta}(\mathbf{r}) \in \mathbb{R}^{n \times m}$ are straightforwardly defined from (5):

$$
\begin{aligned}
\mathbf{L}_{r}(\boldsymbol{\theta})_{i, j} & =\left\{\begin{array}{cc}
\text { in-degree of } i \text {-th vertex } & \text { if } i=j, \\
-\cos \left(\theta_{i}-\theta_{j}\right), & \text { if } i \neq j, j \rightarrow i \\
\text { otherwise }
\end{array}\right. \\
\mathbf{L}_{\theta}(\mathbf{r})_{i, k} & =\left\{\begin{array}{cc}
r_{j} / r_{i} & \text { if } k \text {-th directed edge in } \mathbf{B} \text { is } j \rightarrow i \\
0 & \text { otherwise }
\end{array}\right.
\end{aligned}
$$

where $j \rightarrow i$ denotes a directed edge from the vertex $j$ to $i$.

Remark 1: On a balanced digraph, the $\mathbf{L}_{r}(\boldsymbol{\theta})+\mathbf{L}_{r}^{T}(\boldsymbol{\theta})$ is uniformly positive semi-definite regardless of $\boldsymbol{\theta}$. If $\mathbf{B}^{T} \boldsymbol{\theta}=$ $\mathbf{0}$ (i.e., synchronization of $\boldsymbol{\theta}), \mathbf{L}_{r}(\boldsymbol{\theta})$ reduces to the graph Laplacian $\mathbf{L}_{G}$ from (4). On the other hand, if $\mathbf{B}^{T} \mathbf{r}=\mathbf{0}$, (i.e., $\left.r_{1}=r_{2}=\cdots=r_{n}\right), \mathbf{L}_{\theta}(\mathbf{r})$ reduces to a constant matrix $\mathbf{L}_{\theta}(\mathbf{1})$ that satisfies $\mathbf{L}_{G}=\mathbf{L}_{\theta}(\mathbf{1}) \mathbf{B}^{T}$ on an arbitrary directed graph. 
In such a case, the dynamics of $\boldsymbol{\theta}$ in (7) reduces to a generalized Kuramoto model [6], [7], [8] on an arbitrary digraph with a uniform time-varying frequency $\omega(t)$ :

$$
\dot{\boldsymbol{\theta}}=\omega(t) \mathbf{1}-k \mathbf{L}_{\theta}(\mathbf{1}) \sin \left(\mathbf{B}^{T} \boldsymbol{\theta}\right)
$$

where $\mathbf{L}_{\theta}$ 's $(i, j)$ element is 1 if the $j$-th directed edge enters the $i$-th vertex, and 0 otherwise.

In essence, the stability proof of coupled Hopf oscillators is more involved than that of the Kuramoto model in (9) due to the nonlinear coupling gains of $\mathbf{L}_{r}(\boldsymbol{\theta})$ and $\mathbf{L}_{\theta}(\mathbf{r})$.

Remark 2: For a directed ring (cycle) graph, we can verify

$\mathbf{L}_{r}(\boldsymbol{\theta})=\left[\begin{array}{ccccc}1 & -\cos \left(\theta_{1}-\theta_{2}\right) & 0 & \cdots & 0 \\ 0 & 1 & -\cos \left(\theta_{2}-\theta_{3}\right) & \cdots & 0 \\ \vdots & \vdots & \vdots & \ddots & \vdots \\ -\cos \left(\dot{\theta}_{n}-\theta_{1}\right) & 0 & \cdots & 0 & 1\end{array}\right]$

$\mathbf{B}^{T}=\left[\begin{array}{ccccc}1 & -1 & 0 & \cdots & 0 \\ 0 & 1 & -1 & \cdots & 0 \\ \vdots & \vdots & \vdots & \ddots & \vdots \\ -1 & 0 & \cdots & 0 & 1\end{array}\right], \mathbf{L}_{\theta}(\mathbf{r})=\left[\begin{array}{ccccc}r_{2} / r_{1} & 0 & 0 & \cdots & 0 \\ 0 & r_{3} / r_{2} & 0 & \cdots & 0 \\ \vdots & \vdots & \vdots & \ddots & \vdots \\ 0 & 0 & \cdots & 0 & r_{1} / r_{n}\end{array}\right]$

where $\mathbf{B}$ and $\mathbf{L}_{\theta}(\mathbf{r})$ are $n \times n$ square matrices for a directed cycle, in particular, $\mathbf{L}_{G}=\mathbf{B}^{T}$. Among many possible choices, we choose the $\mathbf{B}$ matrix in (10) such that the corresponding $\mathbf{L}_{\theta}(\mathbf{r})$ is a diagonal matrix. We exploit these properties to simplify our stability proofs.

Example 3: The exponential synchronization of two oscillators $(n=2)$ sheds light on the hierarchical coupling of the radius and phase dynamics. For a bidirectional coupling, (7) can be written as $\dot{\theta}_{1}-\dot{\theta}_{2}=-k\left(r_{2} / r_{1}+r_{1} / r_{2}\right) \sin \left(\theta_{1}-\right.$ $\left.\theta_{2}\right)$. By the result of Example 1, $\theta_{1}-\theta_{2}$ exponentially tends to zero from any $\theta_{1}(0)-\theta_{2}(0) \neq \pm \pi$ and $\mathbf{r}>0$. If $\theta_{1}=\theta_{2}$, we can construct the following virtual y system from (6)

$$
\dot{\mathbf{y}}=-\frac{\lambda}{\rho^{2}} \mathbf{D}(\mathbf{r}, \rho)(\mathbf{y}-\rho \mathbf{1})-k\left[\begin{array}{cc}
1 & -1 \\
-1 & 1
\end{array}\right] \mathbf{y}
$$

which has two particular solutions $\mathbf{y}=\mathbf{r}$ and $\mathbf{y}=(\rho ; \rho)$. Since $\mathbf{y}$ is contracting $(\delta \mathbf{y} \rightarrow \mathbf{0})$ with $\mathbf{D}(\mathbf{r}, \rho)>0$ and $k>0$, we conclude $r_{1} \rightarrow \rho$ and $r_{2} \rightarrow \rho$. If there is only a directed coupling, the proof straightforwardly follows with $\mathbf{L}_{r}(\boldsymbol{\theta})+\mathbf{L}_{r}^{T}(\boldsymbol{\theta})$.

We summarize the prior results in [3], [4], [5] that showed a contraction property when the coupling is stronger than the convergence rate of the uncoupled Hopf oscillator.

Theorem 1: The Hopf oscillators on a balanced digraph given in (2) globally exponentially synchronize with the prescribed phase difference $\phi_{i j}$ if $k \hat{\lambda}_{1}\left(\left(\mathbf{L}_{G}+\mathbf{L}_{G}^{T}\right) / 2\right)>\lambda$ where the Laplacian matrix $\mathbf{L}_{G}$ is obtained by setting zero phase differences $\phi_{i j}=0$ and the same desired radius $\rho_{i}=\rho_{j}$, as in (4). Also, $\hat{\lambda}_{1}(\cdot)$ is the minimum nonzero eigenvalue of $(\cdot)$ while $\lambda$ is a positive convergence rate of the Hopf oscillator.

Proof: See [4] for the proof based on [5]. In essence, (2) can be written as $\{\dot{\mathbf{z}}\}=\left\{\mathbf{f}\left(\mathbf{z}, t ; \rho_{1}\right)\right\}-k \mathbf{L}_{G} \otimes \mathbf{I}_{2}\{\mathbf{z}\}$ with $\{\mathbf{z}\}=\mathbf{T}(\boldsymbol{\phi}, \boldsymbol{\rho})\{\mathbf{x}\}$ by using the circular symmetric property of the Hopf oscillator discussed in Sect. I.
Remark 3: It should be noted that Theorem 1 indeed indicates a truly global convergence result, implying synchronization even from $\theta_{i}(0)-\theta_{j}(0)= \pm \pi$, where the coupled oscillators experience a death of oscillation. Nonetheless, they synchronize to a single non-oscillatory trajectory.

Remark 4: We will show that Theorem 1 is too conservative for global synchronization. For example, two coupled Hopf oscillators would require $k>\lambda / 2$ from Theorem 1 . In contrast, we showed above that $k>0$ is the sufficient and necessary condition of almost global synchronization except on $\theta_{i}(0)-\theta_{j}(0)= \pm \pi$ and $\mathbf{r}(0) \neq \alpha \mathbf{1}, \exists \alpha$, without the need for computing $\hat{\lambda}_{1}\left(\left(\mathbf{L}_{G}+\mathbf{L}_{G}^{T}\right) / 2\right)$. We will derive such a result for a network of an arbitrary size in Sect. III.

\section{Main Results: Almost Global Stability ANALYSIS}

We prove that both $\mathbf{r}$ and $\boldsymbol{\theta}$ almost globally asymptotically synchronize for any $k>0$ on a balanced digraph. The novelty of the proofs here is that we exploit the diagonal matrix $\mathbf{L}_{\theta}(\mathbf{r})$ of each cyclic sub-graph by decomposing a balanced graph into multiple directed cycles.

Theorem 2: Despite the nonlinearly coupled $r_{i}$ and $\theta_{i}$ in $\mathbf{L}_{r}(\boldsymbol{\theta})$ and $\mathbf{L}_{\theta}(\mathbf{r})$, the dynamics of $\mathbf{r}$ in (6) is partially contracting on a balanced digraph, regardless of $\boldsymbol{\theta}$ for $k>0, \forall t$. Furthermore, if $\mathbf{B} \boldsymbol{\theta}$ becomes a constant vector, $\mathbf{r}$ becomes a constant vector exponentially.

Proof: We construct the virtual $\mathbf{y}$-system that has $\mathbf{y}=\mathbf{r}$ as one particular solution from (6)

$$
\dot{\mathbf{y}}=\left(-\frac{\lambda}{\rho^{2}} \mathbf{D}(\mathbf{r}, \rho)-k \mathbf{L}_{r}(\boldsymbol{\theta})\right)(\mathbf{y}-\rho \mathbf{1})-k \rho \mathbf{L}_{r}(\boldsymbol{\theta}) \mathbf{1}
$$

where $\mathbf{D}(\mathbf{r}, \rho)=\operatorname{diag}\left(r_{1}\left(r_{1}+\rho\right), \cdots, r_{n}\left(r_{n}+\rho\right)\right)>0$.

The virtual system of $\mathbf{y}$ is contracting regardless of any $\boldsymbol{\theta}(t)$ and $\mathbf{r}(t)$ since " $-\lambda / \rho^{2} \mathbf{D}(\mathbf{r}, \rho)-k\left(\mathbf{L}_{r}(\boldsymbol{\theta})+\mathbf{L}_{r}(\boldsymbol{\theta})^{T}\right) / 2$ " is uniformly negative on a balanced graph. From Lemmas 1 and $2, \mathbf{y}=\mathbf{r}$ is contracting. In contrast with the uncoupled case, Theorem 2 indicates that $\mathbf{y}=\rho \mathbf{1}$ is not a particular solution of (12) unless $\boldsymbol{\theta}$ perfectly synchronizes such that $\mathbf{B}^{T} \boldsymbol{\theta}=\mathbf{0}$, thus $\mathbf{L}_{r}(\boldsymbol{\theta}) \mathbf{1}=\mathbf{0}$. Hence, if $\mathbf{L}_{r}(\boldsymbol{\theta})$ becomes a constant matrix by a constant $\mathbf{B}^{T} \boldsymbol{\theta}$ or $\mathbf{B} \boldsymbol{\theta}$, then $\mathbf{r}$ also converges to a constant vector exponentially fast.

We now focus on proving the synchronization of the phase $\boldsymbol{\theta}$, independently of $\mathbf{r}$, thereby establishing an interesting hierarchical connection between the dynamics of $\mathbf{r}$ and $\boldsymbol{\theta}$. We state the main result of directed cycles first before generalizing to balanced digraphs.

Theorem 3: If the phase $\boldsymbol{\theta}$ on a directed cyclic graph, given by (7), is initially on $\mathbf{B}^{T} \boldsymbol{\theta}(0) \in(-\pi, \pi)^{m}$ and $\mathbf{r}(0) \neq$ $\alpha \mathbf{1}, \exists \alpha$, it remains on $\mathbf{B}^{T} \boldsymbol{\theta} \in(-\pi, \pi)^{m}$ for all future time and asymptotically synchronizes (i.e., $\left.\mathbf{B}^{T} \boldsymbol{\theta} \rightarrow \mathbf{0}\right)$ for any $k>0$.

Proof: On a directed cycle, $\mathbf{L}_{\theta}(\mathbf{r})$ is a diagonal matrix and $\mathbf{B}$ are $n \times n$ square matrices as defined in (10) due to $n=m$. Let us first prove that $\mathbf{B}^{T} \boldsymbol{\theta}$ tends to a constant vector regardless of $\mathbf{r}(t)$. By multiplying (7) by $\mathbf{B}$, we obtain the following gradient-like system

$$
\mathbf{B} \dot{\boldsymbol{\theta}}=-k \mathbf{B L}_{\theta}(\mathbf{r}(t)) \sin \left(\mathbf{B}^{T} \boldsymbol{\theta}\right)=-k \nabla V(\boldsymbol{\theta})
$$


where $V(\boldsymbol{\theta})=2 \sin \left(\mathbf{B}^{T} \boldsymbol{\theta} / 2\right)^{T} \mathbf{L}_{\theta}(\mathbf{r}) \sin \left(\mathbf{B}^{T} \boldsymbol{\theta} / 2\right)$ and we exploited the fact $\mathbf{L}_{\theta}(\mathbf{r})$ is a diagonal matrix in computing $\nabla V(\boldsymbol{\theta})=\left(\partial V / \theta_{1}, \cdots, \partial V / \theta_{n}\right)^{T}=\mathbf{B L}_{\theta}(\mathbf{r}) \sin \left(\mathbf{B}^{T} \boldsymbol{\theta}\right)$.

We take $V(\boldsymbol{\theta})$ as a Lyapunov function and compute its time-derivative $\dot{V}(\boldsymbol{\theta})$ :

$$
\dot{V}=\nabla V(\boldsymbol{\theta})^{T} \dot{\boldsymbol{\theta}}=-\frac{\dot{\boldsymbol{\theta}}^{T} \mathbf{B}^{T} \dot{\boldsymbol{\theta}}}{k}=-\sum_{i \neq j} \frac{\left(\dot{\theta}_{i}-\dot{\theta}_{j}\right)^{2}}{2 k} \leq 0
$$

We can show that $\theta$ tends to one of the equilibria that satisfy $\dot{V}(\boldsymbol{\theta})=0$. Since $\ddot{\boldsymbol{\theta}}$ is bounded from (7), $\ddot{V}(\boldsymbol{\theta})$ is bounded. Also, $\mathbf{B} \dot{\boldsymbol{\theta}}=\mathbf{0}$ is equivalent to $\mathbf{B}^{T} \dot{\boldsymbol{\theta}}=\mathbf{0}$. By Barbalat's lemma [14], $\dot{V} \rightarrow 0$ thus $\mathbf{B} \dot{\boldsymbol{\theta}} \rightarrow \mathbf{0}$ from (14). Hence, $V(\boldsymbol{\theta})$ is non-increasing and $\mathbf{B}^{T} \boldsymbol{\theta}$ remains on $(-\pi, \pi)^{m}$ with $n=m$ for a directed cycle.

From Theorem 2, a constant $\mathbf{B} \boldsymbol{\theta}$ leads exponentially to a constant $\mathbf{r}$ such that $\dot{\mathbf{r}} \rightarrow \mathbf{0}$ and $\frac{d}{d t} \mathbf{L}_{\theta}(\mathbf{r}) \rightarrow \mathbf{0}$. Hence, for simplicity, we now assume $\mathbf{r}$ is a constant vector instead of carrying $\frac{d}{d t} \mathbf{L}_{\theta}(\mathbf{r}) \rightarrow \mathbf{0}$ terms. Then, how can we prove that $\mathbf{B} \boldsymbol{\theta}$ converges to $\mathbf{0}$, not some arbitrary constant? The key idea is to use $\sin \left(\mathbf{B}^{T} \boldsymbol{\theta} / 2\right)$ instead of $\mathbf{B}^{T} \boldsymbol{\theta}$ or $\sin \left(\mathbf{B}^{T} \boldsymbol{\theta}\right)$. We can compute

$$
\begin{aligned}
\frac{d}{d t} \mathbf{L}_{\theta}(\mathbf{r}) & \sin \frac{\mathbf{B}^{T} \boldsymbol{\theta}}{2}=\mathbf{L}_{\theta}(\mathbf{r}) \mathbf{C}_{1}(t) \frac{\mathbf{B}^{T} \dot{\boldsymbol{\theta}}}{2} \\
& =-k \mathbf{L}_{\theta}(\mathbf{r}) \mathbf{C}_{1}(t) \mathbf{B}^{T} \mathbf{C}_{1}(t) \mathbf{L}_{\theta}(\mathbf{r}) \sin \frac{\mathbf{B}^{T} \boldsymbol{\theta}}{2}
\end{aligned}
$$

where $\mathbf{C}_{1}(t)=\operatorname{diag}\left(\cos \left(\mathbf{B}^{T} \boldsymbol{\theta} / 2\right)\right)$. We used the commutative property of diagonal matrices $\mathbf{L}_{\theta}(\mathbf{r})$ and $\mathbf{C}_{1}(t)$. Note that $\mathbf{C}_{1}(t)$ is invertible since $\mathbf{B}^{T} \boldsymbol{\theta}$ remains on $(-\pi, \pi)^{n}$.

We construct the virtual y system from (15)

$$
\dot{\mathbf{y}}=-k \mathbf{L}_{\theta}(\mathbf{r}) \mathbf{C}_{1}(t) \mathbf{B}^{T} \mathbf{C}_{1}(t) \mathbf{y}
$$

that has two particular solutions: $\mathbf{y}=\mathbf{L}_{\theta}(\mathbf{r}) \sin \left(\mathbf{B}^{T} \boldsymbol{\theta} / 2\right)$ and $\mathbf{y}=\mathbf{0}$. The initial condition $\mathbf{r}(0) \neq \alpha \mathbf{1}, \exists \alpha$ ensures that each element of $\mathbf{r}$ stays different from (12) and hence avoiding a local equilibrium with $\sin \left(\mathbf{B}^{T} \boldsymbol{\theta}\right)=\gamma \mathbf{1}, \exists \gamma$.

Since $\mathbf{L}_{\theta}(\mathbf{r})>0$, the virtual length $V(\delta \mathbf{y})=$ $\delta \mathbf{y}^{T} \mathbf{L}_{\theta}(\mathbf{r})^{-1} \delta \mathbf{y}$ has its time-derivative as

$$
\dot{V}(\delta \mathbf{y})=-k \delta \mathbf{y}^{T} \mathbf{C}_{1}(t)\left(\mathbf{B}^{T}+\mathbf{B}\right) \mathbf{C}_{1}(t) \delta \mathbf{y} \leq 0
$$

Note that a cyclic digraph yields a positive semidefinite $\mathbf{B}^{T}+\mathbf{B}$ matrix with a single zero eigenvalue and its eigenvector $\mathbf{1}$. Hence, the matrix $\mathbf{C}_{1}(t)\left(\mathbf{B}^{T}+\mathbf{B}\right) \mathbf{C}_{1}(t)$ is also uniformly positive semi-definite for any $\mathbf{C}_{1}(t)$ with a single zero eigenvalue (recall Sylvester's law of inertia [15]). Consequently, by Barbalat's lemma, we conclude that $\dot{V}(\delta \mathbf{y}) \rightarrow$ 0 asymptotically (semi-contracting) and $V(\delta \mathbf{y})$ tends to a lower limit.

However, how can we prove $\delta \mathbf{y} \rightarrow \mathbf{0}$ where classical LaSalle's invariance theorem cannot be applied due to the non-autonomous system in (16)? In the present paper, we apply a higher-order Taylor expansion [12] to exploit the fact that $V(\delta \mathbf{y})$ is tending to a lower limit from (17). We first decompose the eigenvectors $[\mathbf{1} / \sqrt{n}, \mathbf{S}]$ of $\mathbf{B}+\mathbf{B}^{T}$ such that $\mathbf{S}^{T} \mathbf{1}=\mathbf{0}, \mathbf{S S}^{T}+\mathbf{1} \mathbf{1}^{T} / n=\mathbf{I}_{n}$, and $\mathbf{S}^{T}\left(\mathbf{B}+\mathbf{B}^{T}\right) \mathbf{S}=\boldsymbol{\Lambda}$ where $\boldsymbol{\Lambda}$ is a diagonal matrix of $(n-1)$ positive eigenvalues of $\mathbf{B}+\mathbf{B}^{T}$.

Let us define $\delta \mathbf{y}_{1}=\mathbf{1}^{T} / \sqrt{n} \mathbf{C}_{1}(t) \delta \mathbf{y} \in \mathbb{R}^{1}$ and $\delta \mathbf{y}_{2}=$ $\mathbf{S}^{T} \mathbf{C}_{1}(t) \delta \mathbf{y} \in \mathbb{R}^{n-1}$. We can verify the following.

$$
\begin{aligned}
& \text { If } \delta \mathbf{y}_{2} \neq \mathbf{0}, V(\delta \mathbf{y}(t+d t))-V(\delta \mathbf{y}(t)) \\
& =-k \delta \mathbf{y}^{T} \mathbf{C}_{1}(t)\left(\mathbf{B}^{T}+\mathbf{B}\right) \mathbf{C}_{1}(t) \delta \mathbf{y}(d t)+\mathcal{O}\left(d t^{2}\right) \\
& =-k \delta \mathbf{y}_{2}^{T} \mathbf{\Lambda} \delta \mathbf{y}_{2}(d t)+\mathcal{O}\left(d t^{2}\right)
\end{aligned}
$$

while if $\delta \mathbf{y}_{2}=\mathbf{0}$,

$$
\begin{aligned}
& V(\delta \mathbf{y}(t+d t))-V(\delta \mathbf{y}(t)) \\
& =-2 k \delta \mathbf{y}^{T} \dot{\mathbf{C}}_{1}(t)\left(\mathbf{B}^{T}+\mathbf{B}\right) \dot{\mathbf{C}}_{1}(t) \delta \mathbf{y}(d t)^{3} /(3 !)+\mathcal{O}\left(d t^{4}\right) \\
& =-\delta \mathbf{y}_{1}^{T} \mathbf{1}^{T} \mathbf{C}_{1}^{-1} \dot{\mathbf{C}}_{1}\left(\mathbf{B}^{T}+\mathbf{B}\right) \dot{\mathbf{C}}_{1} \mathbf{C}_{1}^{-1} \mathbf{1} \delta \mathbf{y}_{1} \frac{2 k(d t)^{3}}{3 ! n}+\mathcal{O}\left(d t^{4}\right) \\
& =-\delta \mathbf{y}_{1}^{T}\left(\mathbf{1}^{T} \mathbf{C}_{1}^{-1} \dot{\mathbf{C}}_{1} \mathbf{S}\right) \boldsymbol{\Lambda}\left(\mathbf{S}^{T} \dot{\mathbf{C}}_{1} \mathbf{C}_{1}^{-1} \mathbf{1}\right) \delta \mathbf{y}_{1} \frac{2 k(d t)^{3}}{3 ! n}+\mathcal{O}\left(d t^{4}\right)
\end{aligned}
$$

where we used $\left(\mathbf{1 1}^{T} / n+\mathbf{S S}^{T}\right) \mathbf{C}_{1}(t) \delta \mathbf{y}=\mathbf{1} \delta \mathbf{y}_{1} / \sqrt{n}+$ $\mathbf{S} \delta \mathbf{y}_{2}=\mathbf{C}_{1}(t) \delta \mathbf{y}$. If $\delta \mathbf{y}_{2}=\mathbf{0}, \delta \mathbf{y}=\mathbf{C}_{1}^{-1}(t) \mathbf{1} / \sqrt{n} \delta \mathbf{y}_{1}$.

Note that $\mathbf{z}^{T} \boldsymbol{\Lambda} \mathbf{z}>0$ for $\forall \mathbf{z} \neq \mathbf{0} \in \mathbb{R}^{n-1}$ and $\mathbf{z}=$ $\mathbf{S}^{T} \dot{\mathbf{C}}_{1} \mathbf{C}_{1}^{-1} \mathbf{1} \neq \mathbf{0}$ unless all the diagonal entries of $\dot{\mathbf{C}}_{1}$ are zero. Since $\dot{\mathbf{C}}_{1}=\mathbf{0}$ only at $\delta \mathbf{y}=\mathbf{0}$ (i.e., $\mathbf{B}^{T} \boldsymbol{\theta}=\mathbf{0}$ ), we can assume $\left(\mathbf{1}^{T} \mathbf{C}_{1}^{-1} \dot{\mathbf{C}}_{1} \mathbf{S}\right) \boldsymbol{\Lambda}\left(\mathbf{S}^{T} \dot{\mathbf{C}}_{1} \mathbf{C}_{1}^{-1} \mathbf{1}\right)>0$.

From (18) and (19), we can conclude that both $\delta \mathbf{y}_{1}$ and $\delta \mathbf{y}_{2}$ should tend to zero, in order for $V(\delta \mathbf{y})$ to tend to a lower limit. This in turn implies $\delta \mathbf{y} \rightarrow \mathbf{0}$. From two particular solutions of (16), we can conclude that $\mathbf{L}_{\theta}(\mathbf{r}) \sin \left(\mathbf{B}^{T} \boldsymbol{\theta} / 2\right)$ tends to zero asymptotically and almost globally on $\mathbf{B}^{T} \boldsymbol{\theta} \in$ $(-\pi, \pi)^{m}$. This implies $\left(\theta_{i}-\theta_{j}\right) / 2 \rightarrow p \pi$ with $p=0, \pm 1$ while $\left(\theta_{i}-\theta_{j}\right)$ is only defined on $[-\pi, \pi]$ (recall modulo $2 \pi$ ). Hence, we conclude that $\theta_{i} \rightarrow \theta_{j}$. This completes the proof of the almost global phase $(\boldsymbol{\theta})$ synchronization without the synchronization of $\mathbf{r}(t)$. Note that we proved the convergence of $\mathbf{B}^{T} \boldsymbol{\theta} / 2$ not $\mathbf{B}^{T} \boldsymbol{\theta}$ so that we eliminated convergence to $\pm \pi$.

We can extend Theorem 3 to bidirectional rings (Corollary 1) and balanced digraphs (Corollary 2) by decomposing each balanced digraph into multiple directed cycles as shown in Fig. 1.

Corollary 1: The phase vector $\boldsymbol{\theta}$ on a bidirectional ring in (7), whose initial differences $\theta_{i}(0)-\theta_{j}(0)$ are not $\pm \pi$, synchronizes (i.e., $\mathbf{B}^{T} \boldsymbol{\theta} \rightarrow \mathbf{0}$ ) for any positive coupling gain $k>0$.

Proof: For a bidirectional ring, the dynamics of $\boldsymbol{\theta}$ in (7) can be written as

$$
\begin{aligned}
\dot{\boldsymbol{\theta}} & =\omega(t) \mathbf{1}-k \mathbf{L}_{\theta}(\mathbf{r}) \sin \left(\mathbf{B}^{T} \boldsymbol{\theta}\right) \\
& =\omega(t) \mathbf{1}-k \mathbf{L}_{\theta 1}(\mathbf{r}) \sin \left(\mathbf{B}_{1}^{T} \boldsymbol{\theta}\right)-k \mathbf{L}_{\theta 2}(\mathbf{r}) \sin \left(\mathbf{B}_{1} \boldsymbol{\theta}\right)
\end{aligned}
$$

where $\mathbf{B}=\mathbf{B}_{1}+\mathbf{B}_{1}^{T}$ and $\mathbf{B}_{1}$ is the same $\mathbf{B}$ of a directed cycle defined in (10). Also, $\mathbf{L}_{\theta}(\mathbf{r})$ is no longer diagonal, whereas $\mathbf{L}_{\theta 1}(\mathbf{r})=\operatorname{diag}\left(r_{2} / r_{1}, r_{3} / r_{2}, \cdots, r_{1} / r_{n}\right)$ is the same diagonal matrix as in the directional cycle case and $\mathbf{L}_{\theta 2}(\mathbf{r})=\operatorname{diag}\left(r_{n} / r_{1}, r_{1} / r_{2}, \cdots, r_{n-1} / r_{n}\right)$. Notice that we expressed the equation by decomposing into two directed cycles of diagonal matrices $\mathbf{L}_{\theta 1}$ and $\mathbf{L}_{\theta 2}$ in order to use the proofs in Theorem 3 (see Fig. 1). Similar to Theorem 3, we 


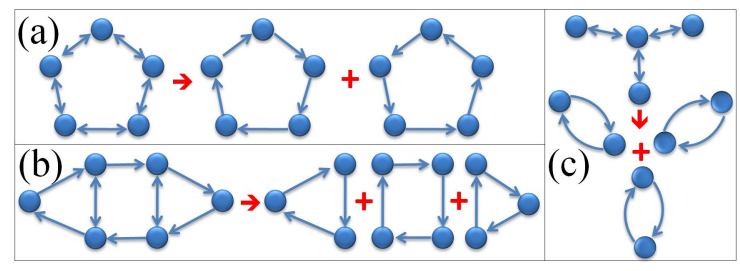

Fig. 1. Cyclic decomposition: (a,c) bi-directional graphs and (b) balanced digraph with directed edges.

can prove that $\mathbf{r}$ tends to a constant vector by taking $V(\Theta)$ with $\Theta=(\boldsymbol{\theta} ; \boldsymbol{\theta})$ :

$$
V(\Theta)=2\left[\begin{array}{c}
\sin \left(\mathbf{B}_{1}^{T} \boldsymbol{\theta} / 2\right) \\
\sin \left(\mathbf{B}_{1} \boldsymbol{\theta} / 2\right)
\end{array}\right]^{T}\left[\begin{array}{cc}
\mathbf{L}_{\theta 1}(\mathbf{r}) & \mathbf{0} \\
\mathbf{0} & \mathbf{L}_{\theta 2}(\mathbf{r})
\end{array}\right]\left[\begin{array}{c}
\sin \left(\mathbf{B}_{1}^{T} \boldsymbol{\theta} / 2\right) \\
\sin \left(\mathbf{B}_{1} \boldsymbol{\theta} / 2\right)
\end{array}\right]
$$

We can compute the time-derivative $\dot{V}(\Theta)$ :

$$
\dot{V}=\nabla V^{T}\left(\begin{array}{c}
\dot{\boldsymbol{\theta}} \\
\dot{\boldsymbol{\theta}}
\end{array}\right)=-\frac{1}{k}\left(\begin{array}{c}
\dot{\boldsymbol{\theta}} \\
\dot{\boldsymbol{\theta}}
\end{array}\right)^{T}\left[\begin{array}{cc}
\mathbf{B}_{1}^{T} & \mathbf{0} \\
\mathbf{0} & \mathbf{B}_{1}
\end{array}\right]\left(\begin{array}{c}
\dot{\boldsymbol{\theta}} \\
\dot{\boldsymbol{\theta}}
\end{array}\right) \leq 0
$$

Hence, we can show $\mathbf{B}_{1} \dot{\boldsymbol{\theta}} \rightarrow 0$, hence $\dot{\mathbf{r}} \rightarrow \mathbf{0}$ by Theorem 2 . Similar to (15), we can obtain

$$
\begin{aligned}
\frac{d}{d t} \mathbf{L}_{\theta 1} \sin \frac{\mathbf{B}_{1}^{T} \boldsymbol{\theta}}{2}= & -k \mathbf{L}_{\theta 1} \mathbf{C}_{1} \mathbf{B}_{1}^{T} \mathbf{L}_{\theta 1} \mathbf{C}_{1} \sin \frac{\mathbf{B}_{1}^{T} \boldsymbol{\theta}}{2} \\
& -k \mathbf{L}_{\theta 1} \mathbf{C}_{1} \mathbf{B}_{1}^{T} \mathbf{L}_{\theta 2} \mathbf{C}_{2} \sin \frac{\mathbf{B}_{1} \boldsymbol{\theta}}{2} \\
\frac{d}{d t} \mathbf{L}_{\theta 2} \sin \frac{\mathbf{B}_{1} \boldsymbol{\theta}}{2}= & -k \mathbf{L}_{\theta 2} \mathbf{C}_{2} \mathbf{B}_{1} \mathbf{L}_{\theta 1} \mathbf{C}_{1} \sin \frac{\mathbf{B}_{1}^{T} \boldsymbol{\theta}}{2} \\
& -k \mathbf{L}_{\theta 2} \mathbf{C}_{2} \mathbf{B}_{1} \mathbf{L}_{\theta 2} \mathbf{C}_{2} \sin \frac{\mathbf{B}_{1} \boldsymbol{\theta}}{2}
\end{aligned}
$$

where $\mathbf{C}_{1}(t)=\operatorname{diag}\left(\cos \left(\mathbf{B}_{1}^{T} \boldsymbol{\theta}(t) / 2\right)\right)$ and $\mathbf{C}_{2}(t)=$ $\operatorname{diag}\left(\cos \left(\mathbf{B}_{1} \boldsymbol{\theta}(t) / 2\right)\right)$.

We can construct the following virtual system of $\mathbf{x}$ and $\mathbf{y}$

$$
\frac{d}{d t}\left(\begin{array}{l}
\mathbf{x} \\
\mathbf{y}
\end{array}\right)=-k\left[\begin{array}{cccc}
\mathbf{L}_{\theta 1} \mathbf{C}_{1} \mathbf{B}_{1}^{T} \mathbf{C}_{1} & \mathbf{L}_{\theta 1} \mathbf{C}_{1} \mathbf{B}_{1}^{T} \mathbf{C}_{2} \\
\mathbf{L}_{\theta 2} \mathbf{C}_{2} \mathbf{B}_{1} \mathbf{C}_{1} & \mathbf{L}_{\theta 2} \mathbf{C}_{2} \mathbf{B}_{1} \mathbf{C}_{2}
\end{array}\right]\left(\begin{array}{l}
\mathbf{x} \\
\mathbf{y}
\end{array}\right)
$$

which has $\left(\mathbf{x}=\mathbf{L}_{\theta 1} \sin \frac{\mathbf{B}_{1}^{T} \boldsymbol{\theta}}{2}, \mathbf{y}=\mathbf{L}_{\theta 2} \sin \frac{\mathbf{B}_{1} \boldsymbol{\theta}}{2}\right)$ and $(\mathbf{x}=$ $\mathbf{0}, \mathbf{y}=\mathbf{0}$ ) as particular solutions.

We can compute the rate change of the virtual length using the metric $\operatorname{diag}\left(\mathbf{L}_{\theta 1}^{-1}, \mathbf{L}_{\theta 2}^{-1}\right)$ as

$$
\frac{d}{d t}\left(\begin{array}{l}
\delta \mathbf{x} \\
\delta \mathbf{y}
\end{array}\right)^{T}\left[\begin{array}{cc}
\mathbf{L}_{\theta 1}^{-1} & \mathbf{0} \\
\mathbf{0} & \mathbf{L}_{\theta 2}^{-1}
\end{array}\right]\left(\begin{array}{l}
\delta \mathbf{x} \\
\delta \mathbf{y}
\end{array}\right)
$$

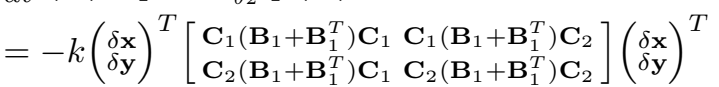

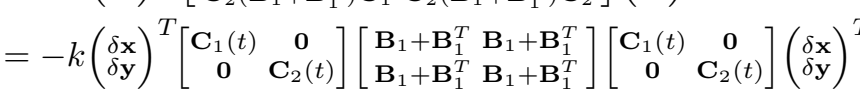

Equation (24) is negative semi-definite with $k>0$ regardless of the signs of $\mathbf{C}_{1}(t)$ and $\mathbf{C}_{2}(t)$. Hence, the system is semi-contracting. By Barbalat's lemma and the high-order Taylor expansion, similar to Theorem $3, \delta \mathbf{x} \rightarrow \mathbf{0}$ and $\delta \mathbf{y} \rightarrow \mathbf{0}$. Hence, all the solutions asymptotically tend to each other. As a result, $\sin \left(\mathbf{B}_{1}^{T} \boldsymbol{\theta} / 2\right) \rightarrow \mathbf{0}$ asymptotically, thus $\mathbf{B}_{1}^{T} \boldsymbol{\theta} \rightarrow \mathbf{0}$.

We can now straightforwardly extend this result to a balanced digraph (see Fig. 1).
Corollary 2: The phase vector $\boldsymbol{\theta}$ on a balanced digraph given in (7), whose initial differences $\theta_{i}(0)-\theta_{j}(0)$ are not $\pm \pi$, synchronizes (i.e., $\mathbf{B}^{T} \boldsymbol{\theta} \rightarrow \mathbf{0}$ ) for any positive coupling gain $k>0$.

Proof: By contradiction, we can prove that every balanced digraph should contain at least one cycle since each node should have the same in-degrees as its out-degrees. Hence, all balanced digraphs with multiple loops can be decomposed into multiple directed cycles, albeit non-unique. This cyclic decomposition holds even for a bi-directional graph without a loop involving more than two vertices (e.g., bidirectional star or tree graphs) since it also belongs to a balanced digraph. In other words, a bidirectional coupling between two vertices can be regarded a single cycle. Hence, we can write (7) as

$$
\dot{\boldsymbol{\theta}}=\omega(t) \mathbf{1}-k \sum_{j=1}^{\ell} \mathbf{L}_{\theta j}(\mathbf{r}) \sin \left(\mathbf{B}_{j}^{T} \boldsymbol{\theta}\right)
$$

where $\ell$ is the number of cyclic subgraphs, $\mathbf{B}_{j}$ is the $j$ th incidence $\mathbf{B}$ matrix of a cyclic subgraph of the original balanced digraph, and $\mathbf{L}_{\theta j}(\mathbf{r})$ is the corresponding diagonal matrix. Note that $\mathbf{B}_{j}$ and $\mathbf{L}_{\theta j}$ have zero row vectors and zero diagonal entries, respectively, where the subgraph does not have vertices. The original incidence matrix $\mathbf{B}$ of the balanced graph can be decomposed as $\mathbf{L}_{G}=\mathbf{L}_{\theta}(\mathbf{1}) \mathbf{B}^{T}=$ $\sum_{j=1}^{\ell} \mathbf{L}_{\theta j}(\mathbf{1}) \mathbf{B}_{j}^{T}=\sum_{j=1}^{\ell} \mathbf{B}_{j}^{T}$, where $\mathbf{L}_{G}$ is the graph Laplacian of a balanced digraph. The rest of the proof follows the proof of Corollary 1 by constructing the dynamics of a stacked vector $\left(\mathbf{L}_{\theta 1} \sin \frac{\mathbf{B}_{1}^{T} \boldsymbol{\theta}}{2} ; \cdots ; \mathbf{L}_{\theta n} \sin \frac{\mathbf{B}_{n}^{T} \boldsymbol{\theta}}{2}\right)$.

We now combine the results from Theorems 2, 3, and Corollaries 1, 2.

Theorem 4: From any initial condition of $r_{i}(0) \neq 0$, $\mathbf{r}(0) \neq \alpha \mathbf{1}, \exists \alpha$ and $\theta_{i}(0)-\theta_{j}(0) \neq \pm \pi$ for all $i, j$, the coupled Hopf oscillators on a balanced digraph, given in (4) and (5), asymptotically synchronize $\left(\theta_{i}=\theta_{j}\right)$, and converge to the same radius $\rho$ for $k>0$.

Proof: If $\theta_{i}=\theta_{j}$ from Theorem 3, the $\mathbf{L}_{r}(\boldsymbol{\theta})$ matrix of nonlinear elements becomes the constant graph Laplacian matrix such that $\mathbf{L}_{r}(\boldsymbol{\theta})=\mathbf{L}_{G}$. We find that the virtual dynamics of radii in (12) reduced to $\frac{d}{d t}(\mathbf{y}-\rho \mathbf{1})=$ $-\lambda / \rho^{2} \mathbf{D}(\mathbf{r}, \rho)(\mathbf{y}-\rho \mathbf{1})-k \mathbf{L}_{G}(\mathbf{y}-\rho \mathbf{1})$, which has two particular solutions $\mathbf{y}=\mathbf{r}$ and $\mathbf{y}=\rho \mathbf{1}$. Note that $\mathbf{D}(\mathbf{r}, \rho)>$ 0 is defined in (6) and we used $\mathbf{L}_{G} \rho \mathbf{1}=\mathbf{0}$. This $\mathbf{y}$-system is contracting $(\delta \mathbf{y} \rightarrow \mathbf{0})$ for any $\mathbf{r}$, since " $-\lambda / \rho^{2} \mathbf{D}(\mathbf{r}, \rho)-$ $k\left(\mathbf{L}_{r}+\mathbf{L}_{r}^{T}\right) / 2$ " is uniformly negative on a balanced graph. Consequently, all the radii of $\mathbf{r}$ tend to the same radius $\rho$ exponentially fast. The combined convergence result of is almost global, but asymptotic (locally exponential) due to asymptotic convergence of $\boldsymbol{\theta}$.

Now we consider a more complex system that has various phase delays, given in (2).

Theorem 5: The coupled Hopf oscillators in (2), which are connected with phase delays and heterogeneous amplitudes on a balanced digraph, asymptotically synchronize on $\left(\mathbf{B}^{T} \boldsymbol{\theta}-\boldsymbol{\phi}\right) \in(-\pi, \pi)^{m}$ and $\mathbf{r}(0) \neq \alpha \mathbf{1}, \exists \alpha$ for $k>0$ in the sense of Definition 1. 
Proof: By dividing the radius dynamics in (3) by $\rho_{i}$ and defining $\mathbf{L}_{\theta}\left(\mathbf{r}^{\prime}\right)$ matrix, we can obtain

$$
\begin{aligned}
\dot{\mathbf{r}}^{\prime} & =-\lambda \mathbf{D}^{\prime}\left(\mathbf{r}^{\prime}\right)\left(\mathbf{r}^{\prime}-\mathbf{1}\right)-k \mathbf{L}_{r}(\boldsymbol{\theta}, \boldsymbol{\phi}) \mathbf{r}^{\prime} \\
\dot{\boldsymbol{\theta}} & =\omega(t) \mathbf{1}-k \mathbf{L}_{\theta}\left(\mathbf{r}^{\prime}\right) \sin \left(\mathbf{B}^{T} \boldsymbol{\theta}-\boldsymbol{\phi}\right)
\end{aligned}
$$

$\mathbf{D}^{\prime}\left(\mathbf{r}^{\prime}\right)=\operatorname{diag}\left(r_{1} / \rho_{1}\left(r_{1} / \rho_{1}+1\right), \cdots, r_{n} / \rho_{n}\left(r_{n} / \rho_{n}+1\right)\right)$ and $\mathbf{r}^{\prime}=\left(r_{1} / \rho_{1}, \cdots, r_{n} / \rho_{n}\right)^{T}$, while $\mathbf{L}_{r}(\boldsymbol{\theta}, \boldsymbol{\phi})$ and $\mathbf{L}_{\theta}\left(\mathbf{r}^{\prime}\right)$ are defined from (3), e.g., $\mathbf{L}_{\theta}\left(\mathbf{r}^{\prime}\right)=$ $\operatorname{diag}\left(\rho_{1} r_{2} /\left(\rho_{2} r_{1}\right), \cdots, \rho_{n} r_{1} /\left(\rho_{1} r_{n}\right)\right)$ for a directed cycle. Also, the phase bias vector $\phi$ of $\phi_{i j}$ for each edge is defined from (3), e.g., $\phi=\left(\phi_{12}, \phi_{23}, \cdots, \phi_{n, 1}\right)^{T}$ for a directed cycle. We can notice that all the previous proofs hold by substituting (a) new $\mathbf{r}^{\prime}$ into $\mathbf{r}$ and (b) $\mathbf{B}^{T} \boldsymbol{\theta}-\boldsymbol{\phi}$ into $\mathbf{B}^{T} \boldsymbol{\theta}$. Hence, by the proofs of Theorems 3 and 4 , we can conclude that $\mathbf{B}^{T} \boldsymbol{\theta} \rightarrow \boldsymbol{\phi}$ on $\left(\mathbf{B}^{T} \boldsymbol{\theta}-\boldsymbol{\phi}\right) \in(-\pi, \pi)^{m}$ (phase synchronization); $r_{i} / \rho_{i} \rightarrow r_{j} / \rho_{j}$ ( $\mathbf{r}$ synchronization); and $\mathbf{r}^{\prime} \rightarrow \mathbf{1}$ (convergence to the limit circles), thereby satisfying Definition 1. These proofs can be extended to a balanced digraph by following cyclic decomposition in Corollary 2 .

Remark 5: Here, $\phi$ is assumed to be a known controllable phase delay in order to define different waveforms (e.g., gaits for locomotion). If unknown $\phi(t)$ is time-varying and $k$ is sufficiently large for exponential synchronization as in Theorem 1, we can apply contraction robustness analysis by regarding $\dot{\phi}$ as a bounded disturbance, as shall be shown in Sect. IV.

Remark 6: The results in Sect. III presented asymptotic synchronization with any positive $k$. If the $k$ gain is sufficiently large as in Theorem 1, Hopf oscillators globally and exponentially synchronize with any $\boldsymbol{\theta} \in \mathbb{T}^{n}$ and $\phi \in \mathbb{T}^{m}$, i.e., $\mathbf{B}^{T} \boldsymbol{\theta} \in[-\pi, \pi]^{m}$ and $\phi \in[-\pi, \pi]^{m}$.

It is now obvious that the proofs in Sect. III hold for uniform Kuramoto oscillators on a balanced graph, hence generalizing the previous results on bi-directional or all-to-all couplings. However, the same radii of the oscillators might lead to the splay state [10]. To get around this problem, we assume that $\left|\theta_{i}\right|<\pi / 2$.

Theorem 6: The Kuramoto model with an identical timevarying frequency $\omega(t)$ on a balanced digraph, given by (9), synchronizes (i.e., $\mathbf{B}^{T} \boldsymbol{\theta} \rightarrow \mathbf{0}$ ), if $k>0$ and $\theta_{i}(0) \in(-\pi / 2+$ $a, \pi / 2+a)$.

Proof: The proof straightforwardly follows the proofs of Theorem 3 and Corollary 2 with constant diagonal matrices $\mathbf{L}_{\theta j}(\mathbf{r})$ for cyclic decomposition of a balanced graph.

\section{SynChronization of Perturbed Heterogeneous Kuramoto Model}

We show here that the synchronization of more complex phase models derived from the Hopf-Kuramoto oscillators can be effectively studied by contraction analysis as in the previous section. For a bidirectional graph (thus balanced), (9) can be generalized with a diagonal matrix of heterogeneous gains $\mathbf{K}(t)$ as well as heterogeneous frequencies $\boldsymbol{\omega}(t)$ and phase delays $\phi(t)$,

$$
\dot{\boldsymbol{\theta}}=\boldsymbol{\omega}(t)-\mathbf{K}(t) \mathbf{B}_{c} \sin \left(\mathbf{B}_{c}^{T} \boldsymbol{\theta}-\boldsymbol{\phi}(t)\right)
$$

where $\mathbf{B}_{c} \in \mathbb{R}^{n \times m / 2}$ is an incidence matrix of each directed link out of bidirectional pairs such that the original incidence matrix is defined as $\mathbf{B}=\left[\mathbf{B}_{c},-\mathbf{B}_{c}\right] \in \mathbb{R}^{n \times m}$. Let us assume that the unknown time-delay vector is realizable such that $\mathbf{1}^{T} \boldsymbol{\phi}=2 \pi p, p=0,1, \cdots$. We state the results for a uniform natural frequency before stating the main result (Theorem 8).

Corollary 3: Consider a network that has more nodes than bidirectional pairs of edges $(n>m / 2)$, e.g., star or tree networks, If $\mathbf{K}(t)>0$ and bounded; $\boldsymbol{\omega}=\omega(t) \mathbf{1}$; and $\boldsymbol{\phi}$ is constant, then $\mathbf{B}_{c}^{T} \boldsymbol{\theta} \rightarrow \boldsymbol{\phi}$ on $\left(\mathbf{B}_{c}^{T} \boldsymbol{\theta}-\boldsymbol{\phi}\right) \in(-\pi, \pi)^{m / 2}$. The convergence result is exponential.

Proof: We can find that the graph Laplacian of bidirectional couplings is $\mathbf{L}_{G}=\mathbf{L}_{\theta}(\mathbf{1}) \mathbf{B}^{T}=\mathbf{B}_{c} \mathbf{B}_{c}^{T}$. Similar to (16), we can obtain $\dot{\mathbf{y}}=-\mathbf{C}_{1}(t) \mathbf{B}_{c}^{T} \mathbf{K}(t) \mathbf{B}_{c} \mathbf{C}_{1}(t) \mathbf{y}$ where $\mathbf{C}_{1}(t)=\operatorname{diag}\left(\cos \left(\left(\mathbf{B}_{c}^{T} \boldsymbol{\theta}-\boldsymbol{\phi}\right) / 2\right)\right)$. Note that " $-\mathbf{C}_{1}(t) \mathbf{B}_{c}^{T} \mathbf{K}(t) \mathbf{B}_{c} \mathbf{C}_{1}(t)$ " is uniformly negative semidefinite on $\left(\mathbf{B}_{c}^{T} \boldsymbol{\theta}-\boldsymbol{\phi}\right) \in(-\pi, \pi)^{m / 2}$, if $m / 2 \geq n$ (e.g., rings, complete graphs, or graphs with loops with other nodes). This is because $\mathbf{B}_{c}^{T} \mathbf{K}(t) \mathbf{B}_{c} \in \mathbb{R}^{m / 2 \times m / 2}$ is uniformly positive semi-definite with the same nullspace as $\mathbf{B}_{c}$. We can conclude asymptotic synchronization $\left(\mathbf{B}_{c}^{T} \boldsymbol{\theta} \rightarrow \boldsymbol{\phi}\right)$ by following the proof of Theorem 3. Also, $\mathbf{B}_{c}^{T} \mathbf{K}(t) \mathbf{B}_{c}$ is uniformly positive definite if $m / 2<n$, since $\mathbf{B}_{c}$ has linearly independent columns. Then, the virtual $\mathbf{y}$-system exponentially synchronizes almost globally on $\left(\mathbf{B}_{c}^{T} \boldsymbol{\theta}-\boldsymbol{\phi}\right) \in(-\pi, \pi)^{m / 2}$ due to Lemmas 1 and 2 .

If $\mathbf{K}=k \mathbf{I}$, we can use "sinc" function [7], which could not be used for Hopf oscillators.

Theorem 7: On $\theta_{i} \in(-\pi / 2+a, \pi / 2+a), \exists a$ and $\left(\mathbf{B}_{c}^{T} \boldsymbol{\theta}-\phi\right) \in(-\pi, \pi)^{m / 2}$, the Kuramoto model (27) with bidirectional couplings, exponentially synchronizes, if $\mathbf{K}=$ $k \mathbf{I}>0 ; \boldsymbol{\omega}=\omega(t) \mathbf{1}$; and $\boldsymbol{\phi}$ is constant.

Proof: If $\mathbf{K}=k \mathbf{I}>0$, we can obtain the $\mathbf{y}$-virtual system by multiplying (27) by $\mathbf{V}_{s}^{T}$

$$
\dot{\mathbf{y}}=-k \mathbf{V}_{s}^{T} \mathbf{B}_{c} \operatorname{diag}\left(\operatorname{sinc}\left(\mathbf{B}_{c}^{T} \boldsymbol{\theta}-\boldsymbol{\phi}\right)\right) \mathbf{B}_{c}^{T} \mathbf{V}_{s} \mathbf{y}
$$

which has two particular solutions: $\mathbf{y}=\mathbf{V}_{s}^{T} \boldsymbol{\theta}-\boldsymbol{\Lambda}^{-1} \mathbf{V}_{s}^{T} \mathbf{B}_{c} \boldsymbol{\phi}$ and $\mathbf{y}=\mathbf{0}$. The matrix $\mathbf{V}_{s}$ is constructed from the orthonormal eigenvectors of $\mathbf{B}_{c} \mathbf{B}_{c}^{T}$, other than $\mathbf{1}$ such that $\mathbf{V}_{s}^{T} \mathbf{1}=\mathbf{0}, \mathbf{V}_{s} \mathbf{V}_{s}^{T}+\mathbf{1 1}^{T} / n=\mathbf{I}_{n}$, and $\mathbf{V}_{s}^{T}\left(\mathbf{B}_{c} \mathbf{B}_{c}^{T}\right) \mathbf{V}_{s}=$ $\boldsymbol{\Lambda}$ with a positive diagonal matrix $\boldsymbol{\Lambda} \in \mathbb{R}^{n-1}$. Since $-k \mathbf{V}_{s}^{T} \mathbf{B}_{c} \operatorname{diag}\left(\operatorname{sinc}\left(\mathbf{B}_{c}^{T} \boldsymbol{\theta}-\boldsymbol{\phi}\right)\right) \mathbf{B}_{c}^{T} \mathbf{V}_{s}$ is uniformly negative definite on $\left(\mathbf{B}_{c}^{T} \boldsymbol{\theta}-\boldsymbol{\phi}\right) \in(-\pi, \pi)^{m / 2}$, by Lemmas 1 and 2, $\mathbf{V}_{s}^{T} \boldsymbol{\theta}$ tends exponentially to $\boldsymbol{\Lambda}^{-1} \mathbf{V}_{s}^{T} \mathbf{B}_{c} \boldsymbol{\phi}$, i.e., $\mathbf{B}_{c}^{T} \boldsymbol{\theta} \rightarrow \phi$.

The main application of Hopf oscillators is generation of phase synchronized oscillatory motions at a common controllable frequency $\omega(t)$ in conjunction with engineered CPGs. However, Kuramoto oscillators are often considered with a non-uniform frequency vector $\boldsymbol{\omega} \neq \omega \mathbf{1}$. In contrast with [7], [8], [9], [16], we further consider a generalized heterogeneous Kuramoto model on an arbitrary bidirectional graph with time-varying vectors $\boldsymbol{\omega}(t)$ and $\phi(t)$ possibly drawn from a random distribution. We characterize the threshold of the coupling strength $k$ guaranteed to yield phase synchronization with a desired bounded phase error. 
Theorem 8: Equation (27) on a bidirectional graph synchronizes on $\theta_{i} \in(-\pi / 2+a, \pi / 2+a), \exists a$ and $\left(\mathbf{B}_{c}^{T} \boldsymbol{\theta}-\boldsymbol{\phi}\right) \in$ $(-\pi, \pi)^{m / 2}$ with bounded phase errors, such that $\mathbf{B}_{c}^{T} \boldsymbol{\theta}$ tends to a ball $\left\|\mathbf{B}_{c}^{T} \boldsymbol{\theta}-\boldsymbol{\phi}(t)\right\| \leq \Phi$, if $\mathbf{K}=k \mathbf{I}$ and

$$
k \geq \sup _{t}\left[\left\|\mathbf{V}_{s}^{T} \boldsymbol{\omega}(t)\right\|+\|\mathbf{d}(t)\|\right]\left\|\mathbf{B}_{c}^{T} \mathbf{V}_{s}\right\| /\left[\sin (\Phi) \hat{\lambda}_{1}\left(\mathbf{L}_{G}\right)\right]
$$

where $\mathbf{d}(t)=\boldsymbol{\Lambda}^{-1} \mathbf{V}_{s}^{T} \mathbf{B}_{c} \dot{\boldsymbol{\phi}}(t)$, and $\mathbf{V}_{s}$ is defined from (28), while $\hat{\lambda}_{1}\left(\mathbf{L}_{G}\right)$ is the smallest nonzero eigenvalue of the graph Laplacian $\mathbf{L}_{G}=\mathbf{B}_{c} \mathbf{B}_{c}^{T}$. Also, $\left\|\mathbf{V}_{s}^{T} \boldsymbol{\omega}(t)\right\|$ and $\|\mathbf{d}(t)\|$ are assumed to be bounded while $\|\cdot\|$ is either 2-norm or $\infty$ norm.

Proof: Similar to (28), we can obtain the following virtual $\mathbf{y}$-system

$\dot{\mathbf{y}}=-k \mathbf{V}_{s}^{T} \mathbf{B}_{c} \operatorname{diag}\left(\operatorname{sinc}\left(\mathbf{B}_{c}^{T} \boldsymbol{\theta}-\boldsymbol{\phi}\right)\right) \mathbf{B}_{c}^{T} \mathbf{V}_{s} \mathbf{y}+\mathbf{V}_{s}^{T} \boldsymbol{\omega}(t)-\mathbf{d}(t)$

where $\mathbf{V}_{s}^{T} \boldsymbol{\omega}-\mathbf{d}(t)$ with $\mathbf{d}=\boldsymbol{\Lambda}^{-1} \mathbf{V}_{s}^{T} \mathbf{B}_{c} \dot{\boldsymbol{\phi}}$ can be viewed as a noisy disturbance or heterogeneous model uncertainty. This problem exemplifies a benefit of exponential convergence. By contraction robustness analysis [5], [11], we can conclude that the distance $R(t)=\int_{P_{1}}^{P_{2}}\|\delta \mathbf{y}\|$ between two arbitrary trajectories, $P_{1}$ and $P_{2}$ of $\mathbf{y}$, will exponentially converge to a ball of

$$
\begin{aligned}
R(t) & \leq \sup _{t}\left[\left\|\mathbf{V}_{s}^{T} \boldsymbol{\omega}(t)-\mathbf{d}(t)\right\|\right] / \beta, \\
\beta & =k \inf _{t} \hat{\lambda}_{\min }\left(\mathbf{V}_{s}^{T} \mathbf{B}_{c} \operatorname{diag}\left(\operatorname{sinc}\left(\mathbf{B}_{c}^{T} \boldsymbol{\theta}-\boldsymbol{\phi}\right)\right) \mathbf{B}_{c}^{T} \mathbf{V}_{s}\right)
\end{aligned}
$$

where the contraction rate $\beta$ is defined from Lemma 1 and $\hat{\lambda}_{\text {min }}(\cdot)$ is the minimum eigenvalue.

Since $\mathbf{y}=\mathbf{V}_{s}^{T} \boldsymbol{\theta}-\boldsymbol{\Lambda}^{-1} \mathbf{V}_{s}^{T} \mathbf{B}_{c} \boldsymbol{\phi}$, we translate the maximum bound $\Phi$ by using $\left\|\mathbf{B}_{c}^{T} \boldsymbol{\theta}-\boldsymbol{\phi}\right\| \leq\left\|\mathbf{B}_{c}^{T} \mathbf{V}_{s}\right\| \| \mathbf{V}_{s}^{T} \boldsymbol{\theta}-$ $\boldsymbol{\Lambda}^{-1} \mathbf{V}_{s}^{T} \mathbf{B}_{c} \boldsymbol{\phi} \| \leq \Phi$. Also, we can find that $\beta=$ $k \operatorname{sinc}\left(\Phi_{\infty}\right) \hat{\lambda}_{1}\left(\mathbf{L}_{G}\right)$ and $\operatorname{sinc}\left(\Phi_{2}\right) \leq \operatorname{sinc}\left(\Phi_{\infty}\right)$. Then, by substituting $\beta$ and $R \leq R_{\max }$ into (31), the condition (29) is obtained. In general, the use of 2 -norm yields a less conservative value of $k$ than $\infty$-norm.

Remark 7: Eqn. (29) yields the sufficient condition for bi-directional graphs even with time-varying $\omega$ and $\phi$, in contrast with the necessary condition in [7], [9] for the Kuramoto model.

Remark 8: Theorem 8 can be used to predict a mean square phase error of (27) with $\phi=0$ and a noisy disturbance, which is expressed as an Itô stochastic differential equation:

$$
d \boldsymbol{\theta}=-k \mathbf{B}_{c} \sin \left(\mathbf{B}_{c}^{T} \boldsymbol{\theta}\right) d t+\boldsymbol{\sigma}(\boldsymbol{\theta}, t) d \mathbf{W}^{d}, \quad \boldsymbol{\theta}(0)=\boldsymbol{\xi}
$$

where $\sigma$ is a matrix-valued function and $\mathbf{W}^{d}$ is a standard $d$ dimensional Wiener process that captures a noisy $\boldsymbol{\omega}(t)$. Also, $\boldsymbol{\xi}$ is a random variable independent of the noise $\mathbf{W}^{d}$. (32) is assumed to satisfy both the Lipschitz condition and the restriction on growth in order to have a unique solution [13]. Note that the noise-free version of $\mathbf{V}_{s}^{T} \boldsymbol{\theta}$ tends to zero from Theorem 8. Then, the stochastic incremental stability [13] between noisy and noise-free trajectories is given by $\mathbb{E}\left(\left\|\mathbf{V}_{s}^{T} \boldsymbol{\theta}(t)\right\|^{2}\right) \leq \sup _{t} \operatorname{tr}\left(\boldsymbol{\sigma}(\boldsymbol{\theta}, t)^{T} \mathbf{V}_{s} \mathbf{V}_{s}^{T} \boldsymbol{\sigma}(\boldsymbol{\theta}, t)\right) /(2 \beta)+$ $\mathbb{E}\left(\left\|\mathbf{V}_{s}^{T} \boldsymbol{\xi}\right\|^{2}\right) e^{-2 \bar{\beta} t}$.

\section{CONCLUSion}

The Andronov-Hopf oscillators are used as the dynamic model of engineered CPGs, which can reduce the complexity associated with control of multiple interacting degrees of freedom. By studying the incremental stability of observerlike virtual systems via partial contraction analysis, we derived new results on almost global synchronization of coupled Hopf oscillators on a balanced digraph with phase delays. Another novelty of the proofs lies in decomposition of a balanced graph into multiple cycles. We showed that any positive coupling gain of coupled Hopf oscillators on a balanced digraph induced almost global synchronization, regardless of the convergence gain of uncoupled oscillators. Furthermore, the incremental stability analysis was applied to a more complicated heterogeneous Kuramoto model. Since the phase dynamic model of Hopf oscillators resembles the Kuramoto model, we can also extend the new synchronization results with time-varying or noisy natural frequencies and phase delays to Hopf oscillators.

\section{REFERENCES}

[1] Z. Chen and T. Iwasaki, "Circulant synthesis of central pattern generators with application to control of rectifier systems," IEEE Trans. Autom. Control, vol. 53, no. 1, pp. 273-286, 2008.

[2] A. J. Ijspeert, A. Crespi, and J.-M. Cabelguen, "Simulation and robotics studies of salamander locmotion: applying neurobiological principles to the control of locomotion in robotics," Neuroinformatics, vol. 3, no. 3, pp. 171-196, 2005.

[3] K. Seo, S.-J. Chung, and J.-J. E. Slotine, "CPG-based control of a turtle-like underwater vehicle," Auton. Robot., vol. 28, no. 3, 2010.

[4] S.-J. Chung and M. Dorothy, "Neurobiologically inspired control of engineered flapping flight," J. Guid., Control, Dyn., vol. 33, no. 2, pp. 440-453, Mar.-Apr. 2010.

[5] Q.-C. Pham and J.-J. E. Slotine, "Stable concurrent synchronization in dynamic system networks," Neural Netw., vol. 20, no. 1, pp. 62-77, Jan. 2007.

[6] S. H. Strogatz, "From Kuramoto to Crawford: exploring the onset of synchronization in populations of coupled nonlinear oscillators," Physica D, vol. 143, 2000.

[7] A. Jadbabaie, N. Motee, and M. Barahona, "On the stability of the Kuramoto model of coupled nonlinear oscillators," in Proc. American Control Conf., 2004.

[8] A. Papachristodoulou and A. Jadbabaie, "Synchonization in oscillator networks with heterogeneous delays, switching topologies and nonlinear dynamics," in Proc. IEEE CDC, San Diego, CA, 2006.

[9] N. Chopra and M. W. Spong, "On exponential synchronization of Kuramoto oscillators," IEEE Trans. Autom. Control, vol. 54, no. 2, pp. 353-357, Feb. 2009.

[10] R. Sepulchre, D. A. Paley, and N. E. Leonard, "Stabilization of planar collective motion: all-to-all communication," IEEE Trans. Autom. Control, vol. 52, no. 5, pp. 811-824, May 2007.

[11] W. Lohmiller and J.-J. E. Slotine, "On contraction analysis for nonlinear systems," Automatica, vol. 34, no. 6, 1998.

[12] W. Wang and J.-J. E. Slotine, "On partial contraction analysis for coupled nonlinear oscillators," Biol. Cybern., vol. 92, no. 1, pp. 3853, Jan. 2005.

[13] Q.-C. Pham, N. Tabareau, and J.-J. E. Slotine, "A contraction theory approach to stochastic incremental stability," IEEE Trans. Autom. Control, vol. 54, no. 4, pp. 816-820, 2009.

[14] J.-J. E. Slotine and W. Li, Applied Nonlinear Control. Prentice Hall, 1991, pp. 122-125.

[15] R. A. Horn and C. R. Johnson, Matrix Analysis. Cambridge University Press, 1985 , pp. 223-238.

[16] F. Dorfler and F. Bullo, "Synchronization and transient stability in power networks and non-uniform Kuramoto oscillators," IEEE Trans. Autom. Control, 2010, submitted. 